\title{
Space-time mapping of historical plague epidemics in modern Osaka, Japan
}

\author{
Tomoki Nakaya $^{\text {a, }}$, Kazumasa Hanaoka ${ }^{b}$, Shohei Nagata ${ }^{a}$ \\ ${ }^{a}$ Graduate School of Environmental Studies, Tohoku University, tomoki.nakaya.c8@tohoku.ac.jp, shohei.nagata.c5@tohoku.ac.jp \\ ${ }^{b}$ College of Letters, Ritsumeikan University, kht27176@fc.ritsumei.ac.jp \\ * Corresponding author
}

Keywords: Historical epidemics, spatial diffusion, social network, spatial epidemiology, space-time statistics

\begin{abstract}
:
In modern times Japan, large-scale epidemics of infectious diseases such as cholera and plague were repeatedly introduced from major cities with ports. Osaka, along with Kobe, is the earliest city in the country to have plague epidemics at that age. To consider the counter measures of such epidemics, epidemic reports were often edited to record the details of the epidemic trends with individual records of infected persons. In case of the plague epidemics in Osaka city, the three-volume set of the Second Osaka Prefectural Report of Plague Epidemics (hereafter, the Plague Epidemic Report) was compiled and published in 1909. According to this report, the plague epidemics prevailed in the city between 1899 and 1900 and between 1905 and 1907. Especially in November 1907, the number of cases exceeded 220 which was the largest number of monthly incidences in the city.
\end{abstract}

This study aims to digitally reconstruct the spatio-temporal sequences of the city-wide historical plague epidemics at the individual level from September 1906 to the end of December 1907, recorded in the Plague Epidemic Report, by using a geographic information system (GIS). We examine the possibilities of visual understanding about the geographical processes of the plague epidemics in the city through space-time mapping of the historical materials of the disease in a GIS environment. The database and visualized space-time features of the epidemics would be useful for theoretical studies of infectious disease epidemiology with spatial dimension, since it is not easy to obtain such detailed individual data of infected persons in a city-wide extent at present days due to privacy protection policies. In addition, this study may highlight the old city structure of Osaka in modern times through the epidemic sequences. This may contribute to the studies of historical geography as well as those of Digital Humanities in a wider context.

We developed the GIS-based space-time database of plague cases as follows. Firstly, we digitally archived the Plague Epidemic Report by scanning the entire pages including Figures and Tables. Secondly, we built a database of cases from the scanned list of plague case lists with their attributes including id number, the date of onset, the reason and date of identification, the disease type, address, location of discovery, occupation, sex, age and others. Thirdly, geographical coordinates of the occurrence location were identified as the points drawn in the 1:20,000 scale maps included in the Plague Epidemic Report with the aid of GIS-based geo-referencing. At the result, the records of 661 cases with the geographical coordinates were established. In addition, we construct other geographical information in the Plague Epidemic Report, such as the aggregated numbers of caught rats carrying the plague by police box regions over the city. It would be interesting to note that the distribution of plague cases is almost consistent with the distribution of the number of infected rats.

Using the spatiotemporal database developed here, we conduct the mapping of plague cases in a space-time cube setting (Figure 1). Each black dot in the figure represents one infected case with the geographical locations and timing (temporal locations) defined by his/her onset day-counts after 13 Sep 1906 when the first case in the list appeared. We enhanced the visualization of space-time point distribution by using space-time kernel density estimation as shown in Figure 2 including two iso-surfaces of high (coloured in red) and middle (coloured in blue) density domains. We developed a tool for ArcGIS Pro (ESRI Inc.) combined with R (R Core Team) to generate the space-time iso-surfaces of densities. The tool enables us to create a web-based interactive digital content of the historical epidemic.

It is noteworthy that the case attribute in the database included relationships with other recorded cases. Figure 2 has the lines showing the relationships between the cases. While most of them are short and almost vertically stand meaning that infections were occurred in a limited geographical extent, there are also long-length lines indicating that local outbreaks at different locations were connected by the person-to-person contacts, possibly reflecting possibly commuting networks at that age. We intend to argue how the plague epidemics spread in the city by associating the space-time sequences of infection with the demographic and socioeconomic attributes of the cases in the context of modern Osaka. 


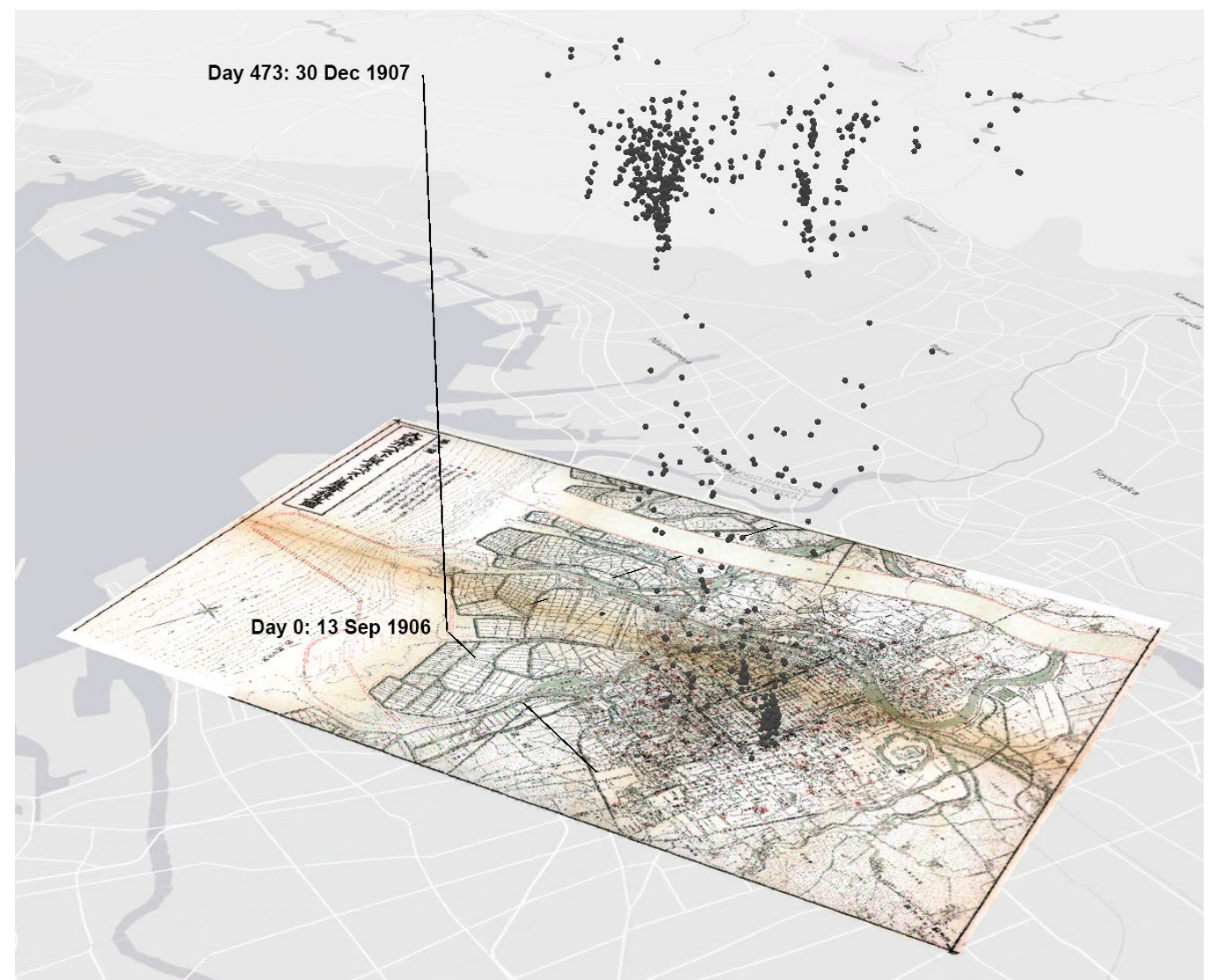

Figure 1 Space-time mapping of plague cases in Osaka city from Sep 1906 to Dec 1907

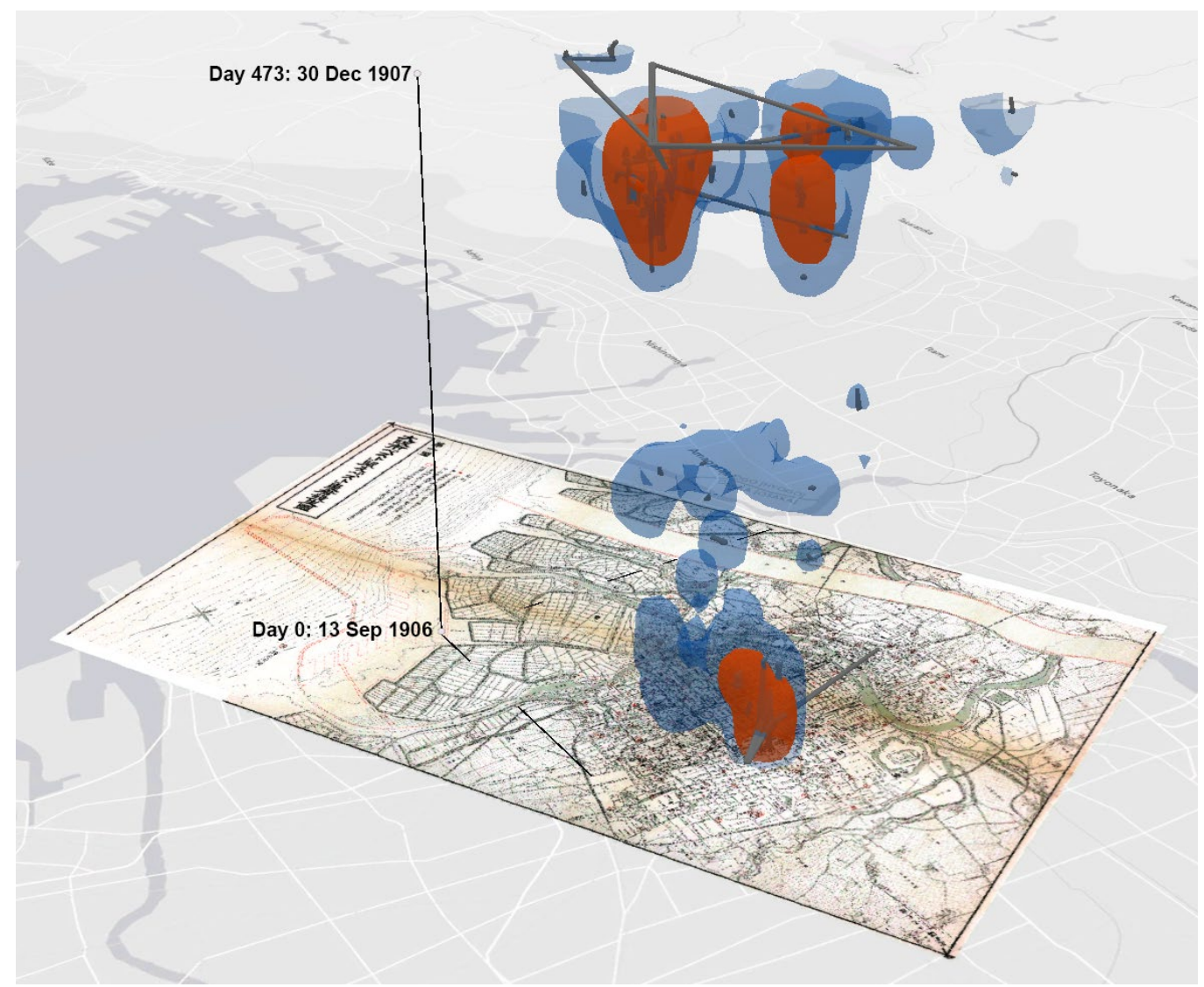

Figure 2. Space-time mapping of high and middle density domains of plague cases and lines corresponding to recorded connections between the cases. 